\title{
Two-stage inflation as a solution to the initial condition problem of hybrid inflation
}

\author{
C. Panagiotakopoulos ${ }^{1}$ and N. Tetradis ${ }^{2}$ \\ ${ }^{1}$ Physics Division, School of Technology, University of Thessaloniki, Thessaloniki 540 06, Greece \\ ${ }^{2}$ CERN, Theory Division, CH-1211, Geneva 23, Switzerland
}

(Received 5 November 1997; published 11 March 1999)

\begin{abstract}
We address the issue of fine-tuning of the initial field configuration that can lead to hybrid inflation in the context of global supersymmetry. This problem is generated by the difference between the energy scale at which the Universe emerges from the Planck era and the inflationary scale implied by the COBE observations: $\quad V^{1 / 4} \sim 10^{-3} m_{\mathrm{Pl}}$. We propose a scenario with two stages of inflation. The first stage, with a typical scale not far from $m_{\mathrm{Pl}}$, occurs "naturally" and provides the necessary homogeneity for the second stage. The latter generates the density perturbations that result in the cosmic microwave background anisotropy observed by COBE. [S0556-2821(99)04704-9]

PACS number(s): $98.80 . \mathrm{Cq}$
\end{abstract}

\section{INTRODUCTION}

The construction of a realistic inflationary scenario is constrained by the following main requirements: (a) A realistic particle physics model must be constructed, which guarantees the presence of a phase where the stress-energy tensor of the Universe is dominated by the potential energy density; (b) this phase must emerge "naturally," so that the initial conditions for the onset of inflation are not fine-tuned; (c) the experimental constraints, resulting mainly from the Cosmic Background Explorer (COBE) observation of the cosmic microwave background anisotropy, must be satisfied.

The most common way of satisfying the first requirement is by constructing a field-theoretical model that predicts an almost flat direction in field space with nonzero potential energy density. A very small slope of the potential along this direction results in the slow rolling of the inflaton field $\sigma$. Inflation stops when the inflaton rolls beyond the point where the flat direction ends. It is crucial that the flatness of the potential in the inflationary trajectory be preserved by quantum (and possible thermal) corrections. A natural implementation of this scenario occurs when hybrid inflation [1] is embedded in the context of supersymmetry [2-9]. Flat directions in the potential appear without fine-tuning and are stable against quantum corrections. An important aspect of the hybrid inflationary scenario is that the part of inflation with observable consequences takes place for values of the inflaton field below the Planck scale [2] where models can be reliably constructed. (Throughout the paper we use the "reduced" Planck scale $m_{\mathrm{Pl}}=M_{\mathrm{Pl}} / \sqrt{8 \pi}, \quad M_{\mathrm{Pl}}$ $=1.2 \times 10^{19} \mathrm{GeV}$.)

A crucial aspect of the problem of initial conditions is whether the presence of inhomogeneities or nonzero time derivatives of the fields prevents the onset of inflation. Previous studies focused on the evolution of spatial or time derivatives of the inflaton field (for a review, see Ref. [10]). For the "new" inflationary scenario $[11,12]$, the influence of such derivatives makes the fine-tuning of the initial conditions necessary for a sufficient amount of inflation to take place $[13,10]$. For chaotic inflation [14], this problem is not severe [10]. In the context of hybrid inflation, the question of initial conditions has been addressed recently [15-17]. In
Ref. [17] it was shown that severe fine-tuning of the initial configuration that will lead to inflation is necessary. This is a consequence of the presence of (one or more) scalar fields coupled to the inflaton and the need to satisfy the observational constraints. We briefly summarize the arguments below.

For hybrid inflation the slow rolling of the inflaton occurs along a valley of the potential. It ends when the valley turns into a ridge and the slow-roll regime terminates, owing to the growth of fluctuations of fields orthogonal to the inflation field. Typically, this point of instability corresponds to a value of the inflaton below the Planck scale. The COBE observation of the cosmic microwave background anisotropy constrains the properties of the model along the inflationary trajectory $[12,18,19]$. On general grounds, one expects the inflationary energy scale $V^{1 / 4}$ (determined by the vacuum energy density during inflation) to be at least two or three orders of magnitude smaller than the Planck scale [20]:

$$
V^{1 / 4} / \epsilon^{1 / 4} \simeq 7 \times 10^{16} \mathrm{GeV}
$$

Here $\epsilon$ is a "slow-roll" parameter [19] that must be much smaller than 1 during inflation. The onset of inflation requires a region of space of a size of a few Hubble lengths where the fields take almost constant values, so that the gradient energy density is negligible compared to the potential energy density. The earliest time at which one could start talking about such regions of space is when the Planck era (during which quantum gravitational fluctuations dominate) ends and classical general relativity starts becoming applicable. The initial energy density is of order $m_{\mathrm{Pl}}^{4}$. For a theory with couplings not much smaller that 1 , the initial field values within each region are expected to be of order $m_{\mathrm{Pl}}$.

Inflation could start at the end of the Planck era, provided that the fields take appropriate values. However, it is most likely that the fields will evolve from some initial values that do not give rise to inflation to different values that do. The difference between the initial energy scale $m_{\mathrm{Pl}}$ of the field evolution and the inflationary scale $V^{1 / 4}$ implies that the fields evolve for a long time before settling down along the inflationary trajectory. The Hubble parameter $H$ sets the scale for the "friction" term in the evolution equations, 
which determines how fast the energy is dissipated through expansion. When the energy density drops much below $m_{\mathrm{Pl}}^{4}$, the smallness of the "friction" term results in a very long evolution, during which the fields oscillate around zero many times. Some of the trajectories eventually settle down in the valley of the potential that produces inflation. However, the sensitivity to the initial conditions is high because of the long evolution. A slight variation of the initial field values separates inflationary trajectories from trajectories that lead to the minima of the potential, where inflation does not occur.

The implications for the initial configuration that will lead to the onset of inflation are severe. It was shown in Ref. [17] that, for the prototype model of hybrid inflation [1] with a scale consistent with the COBE observations, the most favorable area of inflationary initial conditions is a thin strip of width $10^{-5} m_{\mathrm{Pl}}$ around the $\sigma$ axis. Throughout a region of space of a size of the order of the Hubble length (which initially is $\sim m_{\mathrm{Pl}}^{-1}$ ), the initial value of the field orthogonal to the inflation must be zero with an accuracy $10^{-5} m_{\mathrm{Pl}}$. This should be compared to the natural scale of the initial fluctuations of the fields, which is of order $m_{\mathrm{Pl}}$. If this condition of extreme homogeneity is not satisfied, the fields in different parts of the original space region will evolve towards very different values. In one part they may end up in the valley along the $\sigma$ axis, while in another they may settle at the minima of the potential. Before inflation sets in, the size of the space regions shrinks compared to the Hubble distance. As a result, large inhomogeneities are expected at scales smaller than $\sim H^{-1}$ when the evolution of the fields finally slows down. These will prevent the onset of inflation. The fine-tuning of the initial configuration must be increased by several orders of magnitude if the initial time derivatives of the fields are nonzero.

In Ref. [17] the evolution of the scale factor $R$ relative to the Hubble parameter was also studied, starting from an initial value $R_{0} \sim H_{0}^{-1}$. At the onset of inflation, $R$ was found to be smaller than $H^{-1}$ typically by a factor of order 10-100. This implies that the initial homogeneous region should extend far beyond a few initial Hubble lengths for this region to inflate.

In this paper we suggest a simple resolution of the issue of fine-tuning described above. The origin of the problem lies in the difference between the inflationary scale of Eq. (1.1) implied by the COBE observations and the Planck scale. This difference has two consequences.

(a) The "friction" term, proportional to $H$, in the evolution equations becomes very small when the energy density drops far below the Planck scale. As a result, a long evolution takes place before the system settles down along a flat direction or at the minima of the potential. This generates very high sensitivity to the initial conditions and requires extreme homogeneity of the initial configuration, as we explained above.

(b) The reduction of $R$ relative to $H^{-1}$ during the preinflationary evolution implies that this extreme homogeneity must be assumed far beyond the initial Hubble length.

The first problem can be resolved if the Hubble parameter stays large until the fields settle down along the flat direction that will eventually produce inflation. The presence of an additional field sector with initial energy density of order $m_{\mathrm{Pl}}^{4}$ can keep $H$ large during this first part of the evolution. The second problem indicates the necessity of a first stage of inflation that will produce a high degree of homogeneity far beyond the Hubble length.

The above considerations have motivated us to envision a scenario with two stages of inflation. The first stage has a typical scale $\sim m_{\mathrm{Pl}}$, occurs "naturally," and provides the homogeneity that is necessary for the second stage. The latter generates the density perturbations that result in the cosmic microwave background anisotropy observed by COBE. Similar ideas have been discussed in Ref. [21] in the context of chaotic inflation. A scenario analogous to ours has been proposed for the resolution of the problem of initial conditions for "new" inflation [22].

In Sec. II we present a quantitative discussion of the finetuning of the initial conditions for hybrid inflation in the context of a globally supersymmetric model. In Sec. III we introduce an extension of the model that allows for an additional first stage of inflation. This resolves the problem of fine-tuning. We conclude by discussing the possible implementation of the idea in the context of supergravity.

\section{ONE-STAGE INFLATION AND FINE-TUNING}

We consider a supersymmetric model described by the superpotential $[2,3]$

$$
W=S\left(-\mu^{2}+\lambda \Phi \Phi\right) .
$$

Here $S, \Phi$, and $\Phi$ are chiral superfields, for which we assume canonical kinetic terms. The above superpotential is the only renormalizable one consistent with a continuous $\mathrm{U}(1) R$ symmetry under which $W \rightarrow e^{i \theta} W, S \rightarrow e^{i \theta} S, \bar{\Phi} \Phi \rightarrow \bar{\Phi} \Phi$. The superfields $\Phi$ and $\bar{\Phi}$ transform under an internal gauge symmetry, which we take to be a U(1) symmetry for simplicity: $\Phi \rightarrow e^{i \omega} \Phi, \bar{\Phi} \rightarrow e^{-i \omega} \bar{\Phi}$. The $S$ superfield is a gauge singlet. The gauge symmetry is spontaneously broken at the scale $\mu / \sqrt{\lambda}$. The potential is given by ${ }^{1}$

$$
V=\left|-\mu^{2}+\lambda \bar{\Phi} \Phi\right|^{2}+\lambda^{2}|S|^{2}\left(|\Phi|^{2}+|\bar{\Phi}|^{2}\right)+D \text { terms. }
$$

Vanishing of the $D$ terms is achieved along the $D$-flat directions where $|\Phi|=|\Phi|$.

Through appropriate gauge and $R$ transformations along the $D$-flat directions, we can bring fields in the form

$$
S=\frac{\sigma}{\sqrt{2}}, \quad \Phi=\bar{\Phi}=\frac{\phi_{1}+i \phi_{2}}{2} .
$$

The real fields $\sigma, \phi_{1}, \phi_{2}$ have canonically normalized kinetic terms. The potential can now be written as

\footnotetext{
${ }^{1}$ We use the same notation for superfields and their scalar components. Also we have interchanged the notation for the inflaton and its orthogonal field with respect to Ref. [17].
} 


$$
\begin{aligned}
V\left(\sigma, \phi_{1}, \phi_{2}\right)= & \mu^{4}-\frac{\lambda}{2} \mu^{2}\left(\phi_{1}^{2}-\phi_{2}^{2}\right)+\frac{\lambda^{2}}{16}\left(\phi_{1}^{2}+\phi_{2}^{2}\right)^{2} \\
& +\frac{\lambda^{2}}{4} \sigma^{2}\left(\phi_{1}^{2}+\phi_{2}^{2}\right) .
\end{aligned}
$$

The vacua are located at $\sigma=0, \phi_{1}^{2}=4 \mu^{2} / \lambda, \phi_{2}=0$. The potential has a flat direction along the $\sigma$ axis with $V\left(\sigma, \phi_{1}\right.$ $\left.=\phi_{2}=0\right)=\mu^{4}$. Along this direction the mass terms of the $\phi_{1,2}$ fields are

$$
\left[M_{\phi}^{2}\right]_{1,2}=\mp \lambda \mu^{2}+\frac{\lambda^{2}}{2} \sigma^{2} .
$$

An instability appears for

$$
\sigma^{2}<\sigma_{\text {ins }}^{2}=\frac{2 \mu^{2}}{\lambda},
$$

which can trigger the growth of the $\phi_{1}$ field.

The flatness of the potential along the $\sigma$ axis is lifted by radiative corrections, resulting from the breaking of supersymmetry by the nonzero value of $V\left(\sigma, \phi_{1}=\phi_{2}=0\right)$. For a $\mathrm{U}(1)$ gauge symmetry and for $\sigma \gg \sigma_{\text {ins }}$, the one-loop contribution to the effective potential along the $\sigma$ axis is given by $[3,16]$

$$
\Delta V(\sigma)=\frac{\lambda^{2}}{16 \pi^{2}} \mu^{4}\left[\ln \left(\frac{\lambda^{2} \sigma^{2}}{2 \Lambda^{2}}\right)+\frac{3}{2}\right]
$$

The precise value of the normalization scale $\Lambda$ is not important for our discussion. The importance of the above contribution for our discussion lies in that it generates a small positive slope along the $\sigma$ axis:

$$
\Delta V^{\prime}(\sigma)=\frac{\lambda^{2}}{8 \pi^{2}} \frac{\mu^{4}}{\sigma} .
$$

We assume that a Robertson-Walker metric is a good approximation for the regions of space with uniform fields that we are considering. The evolution of the fields is given by the standard equations

$$
\begin{array}{r}
\ddot{\sigma}+3 H \dot{\sigma}=-\frac{\partial V\left(\sigma, \phi_{1}, \phi_{2}\right)}{\partial \sigma} \\
\ddot{\phi}_{1,2}+3 H \dot{\phi}_{1,2}=-\frac{\partial V\left(\sigma, \phi_{1}, \phi_{2}\right)}{\partial \phi_{1,2}},
\end{array}
$$

where

$$
H^{2}=\left(\frac{\dot{R}}{R}\right)^{2}=\frac{1}{3 m_{\mathrm{Pl}}^{2}}\left[\frac{1}{2} \dot{\sigma}^{2}+\frac{1}{2} \dot{\phi}_{1}^{2}+\frac{1}{2} \dot{\phi}_{2}^{2}+V\left(\sigma, \phi_{1}, \phi_{2}\right)\right] .
$$

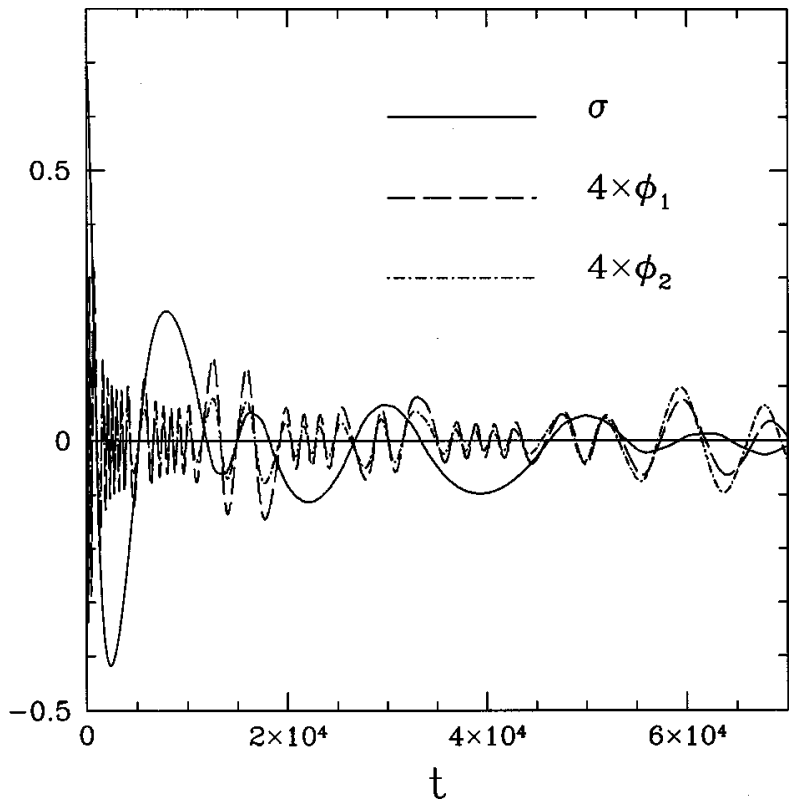

FIG. 1. Evolution of $\sigma, \phi_{1}, \phi_{2}$ (in units of $m_{\mathrm{Pl}}$ ) for the theory of Eq. (2.1) with $\mu / m_{\mathrm{Pl}}=5 \times 10^{-4}, \lambda=0.05$, and initial conditions $\sigma_{0} / m_{\mathrm{Pl}}=0.7,\left[\phi_{1}\right]_{0} / m_{\mathrm{Pl}}=0.1,\left[\phi_{2}\right]_{0} / m_{\mathrm{Pl}}=0.05$.

Inflation can occur in the almost flat direction along the $\sigma$ axis. For $\sigma \gg \sigma_{\text {ins }}{ }^{2}$ the "slow-roll" parameters $\epsilon, \eta$ [19] are small as long as $\sigma / m_{\mathrm{Pl}} \geq \lambda / \sqrt{8 \pi^{2}}$. For $\lambda \gtrsim 10^{-3}$ the spectrum of the adiabatic density perturbations in this inflationary scenario is estimated to be [3]

$$
\delta_{H} \simeq \sqrt{\frac{N_{Q}}{75}} \frac{4}{\lambda}\left(\frac{\mu}{m_{\mathrm{Pl}}}\right)^{2},
$$

where $N_{Q} \simeq 60$ is the number of $e$-foldings of our present horizon scale during inflation. Comparison with the value $\delta_{H}=1.94 \times 10^{-5}$, deduced from the COBE observation of the cosmic microwave background anisotropy, gives

$$
\frac{\mu}{m_{\mathrm{Pl}}} \simeq 2.3 \times 10^{-3} \sqrt{\lambda}
$$

As we have discussed in the Introduction, inflation does not start immediately, as soon as the Universe emerges from the Planck era. An initial evolution of the fields takes place, during which they approach the flat direction and eventually settle on the slow-roll trajectory. A typical example of this part of the evolution is depicted in Fig. 1. We have taken $\lambda=0.05$ and chosen the mass scale $\mu / m_{\mathrm{Pl}}=5 \times 10^{-4}$ near the value implied by the COBE observations. The initial field values are near $m_{\mathrm{Pl}}$, with $\phi_{1}, \phi_{2}$ smaller than $\sigma$, so that the evolution starts near the flat direction $\left(\sigma_{0} / m_{\mathrm{Pl}}=0.7\right.$, $\left.\left[\phi_{1}\right]_{0} / m_{\mathrm{Pl}}=0.1,\left[\phi_{2}\right]_{0} / m_{\mathrm{Pl}}=0.05\right)$. The evolution equations (2.9)-(2.11) have been integrated numerically. The small radiative contribution of Eq. (2.7) has been neglected,

\footnotetext{
${ }^{2}$ Inflation can persist down to $\sigma_{\text {ins }}$ for sufficiently small coupling $\lambda$. For a discussion, see Refs. $[3,16]$.
} 
so that $\phi_{1}=\phi_{2}=0$ corresponds to a completely flat direction. The initial time derivatives of the fields have been set to zero. This is the most favorable situation for the onset of inflation. We observe an initial stage during which the $\sigma$ field decays, while $\phi_{1}$ and $\phi_{2}$ oscillate rapidly around zero. The decay is due to the large initial value of $H$, induced by the large average value of $V$ in Eq. (2.11). When $\sigma$ becomes much smaller than its initial value, $H$ drops significantly and the "friction" term in Eqs. (2.9), (2.10) becomes less important. As a result, the system of fields enters a stage of oscillations around zero, with very slowly decaying amplitudes. This stage lasts for a time larger than the interval depicted in Fig. 1 by several orders of magnitude. Eventually, the system settles down either along the flat direction or at the minima of the potential.

This type of evolution generates high sensitivity to the initial conditions. A slight variation of the initial field values separates inflationary trajectories from trajectories that lead to the minima of the potential, where inflation does not occur. This high sensitivity has severe implications for the initial configuration that will lead to the onset of inflation. For inflation to start, a region of space of a size of a few Hubble lengths is required, in which the fields take almost constant values, so that the gradient energy density is negligible compared to the potential energy density. However, it is not sufficient to assume that such a homogeneous region emerges at the end of the Planck era, since this homogeneity must be maintained during the whole evolution, up to the point where inflation starts. For this to happen, the fields within the region of space should not vary more than the minimal difference between the initial values for a trajectory that eventually leads to inflation and those for a trajectory that leads to the minima of the potential. If this condition of extreme homogeneity is not satisfied, the fields in different parts of the original space region will evolve towards very different values. Before inflation sets in, the size of space regions shrinks with respect to the Hubble distance. As a result, large inhomogeneities will appear at scales smaller than $\sim H^{-1}$ when the evolution of the fields finally slows down, which will prevent the onset of inflation.

It was shown in Ref. [17] that, for the prototype model of hybrid inflation [1] and $\mu \lesssim 10^{-1} m_{\mathrm{Pl}}$, the most favorable area of inflationary initial conditions is a thin strip around the $\sigma$ axis. If the fields start in this area without initial time derivatives, $\sigma$ does not oscillate around zero, but quickly settles along the flat direction. We can obtain a rough estimate of the width of this area if we consider Eq. (2.9) and replace $\phi_{1}^{2}$ and $\phi_{2}^{2}$ by their average values $\left[\phi_{1,2}^{2}\right]_{\mathrm{rms}}$ $\sim\left[\phi_{1,2}\right]_{0}^{2} / 2$ during the evolution. The equation now reads

$$
\ddot{\sigma}+3 H \dot{\sigma}=-\frac{\lambda^{2}}{4}\left(\left[\phi_{1}\right]_{0}^{2}+\left[\phi_{2}\right]_{0}^{2}\right) \sigma .
$$

Two time scales characterize the solutions of this equation. The first one is related to the friction term and is given by $t_{H}^{-1}=3 H / 2$. For $\left[\phi_{1,2}\right]_{0} \ll 2 \mu / \sqrt{\lambda}$ and $\lambda^{2} \sigma^{2}\left(\left[\phi_{1}\right]_{0}^{2}\right.$ $\left.+\left[\phi_{2}\right]_{0}^{2}\right) / 8 \ll \mu^{4}$, we have

$$
t_{H}=\frac{2}{\sqrt{3}} \frac{m_{\mathrm{Pl}}}{\mu^{2}} .
$$

The other time scale is obtained if we neglect the friction term and consider the oscillations of the $\sigma$ field. One-fourth of the period is the typical time for the system to roll to the origin and away from an inflationary solution. It is given by

$$
t_{\mathrm{osc}}=\frac{\pi}{\lambda} \frac{1}{\sqrt{\left[\phi_{1}\right]_{0}^{2}+\left[\phi_{2}\right]_{0}^{2}}} .
$$

Inflation sets in if $t_{\mathrm{osc}} \geq t_{H}$, which gives

$$
\frac{\sqrt{\left[\phi_{1}\right]_{0}^{2}+\left[\phi_{2}\right]_{0}^{2}}}{m_{\mathrm{Pl}}} \lessgtr \frac{\sqrt{3} \pi}{2 \lambda}\left(\frac{\mu}{m_{\mathrm{Pl}}}\right)^{2} \text {. }
$$

We have verified numerically that the above relation gives the correct order of magnitude for the size of the strip around the $\sigma$ axis that leads to inflationary solutions. Our assumptions for the derivation of the above bound break down when $\sigma \gtrsim \sqrt{24 / \pi^{2}} m_{\mathrm{Pl}}=1.6 m_{\mathrm{Pl}}$.

For our choice of $\mu$ and $\lambda$, the initial value of the fields orthogonal to the inflation must be zero with an accuracy $\sim 10^{-5} m_{\mathrm{Pl}}$ throughout a region of space of a size of the order of the Hubble length. This should be compared to the natural scale of the initial fluctuations of the fields, which is of order $m_{\mathrm{Pl}}$. The fine-tuning of the initial configuration must be increased by several orders of magnitude if the initial time derivatives of the fields are nonzero [17]. In Ref. [17] the evolution of the scale factor $R$ relative to the Hubble parameter was also studied, starting from an initial value $R_{0}$ $\sim H_{0}^{-1}$. At the onset of inflation, $R^{-1}$ was found to be smaller than $H^{-1}$ typically by a factor of order 10-100. This implies that the initial homogeneous region should extend far beyond a few initial Hubble lengths for this region to inflate. It is very difficult to calculate a probability distribution for the initial field configurations and thus have a quantitative estimate of the probability of inflation to occur. This requires a reliable description of Planck scale dynamics. For this reason we cannot address the question of whether such a homogeneous initial state is probable or not. The above results, however, indicate that one-stage hybrid inflation does not occur "naturally." In the following section we describe two possible extensions of the model, within which the problem of the fine-tuning of the initial conditions is resolved.

\section{TWO-STAGE INFLATION}

We consider two extensions of the model of the previous section, which permit a two-stage inflation.

\section{A. Chaotic-hybrid model}

The first model is described by the superpotential

$$
W=S\left(-\mu^{2}+\lambda \Phi \Phi\right)+\frac{1}{2} m S^{\prime 2},
$$




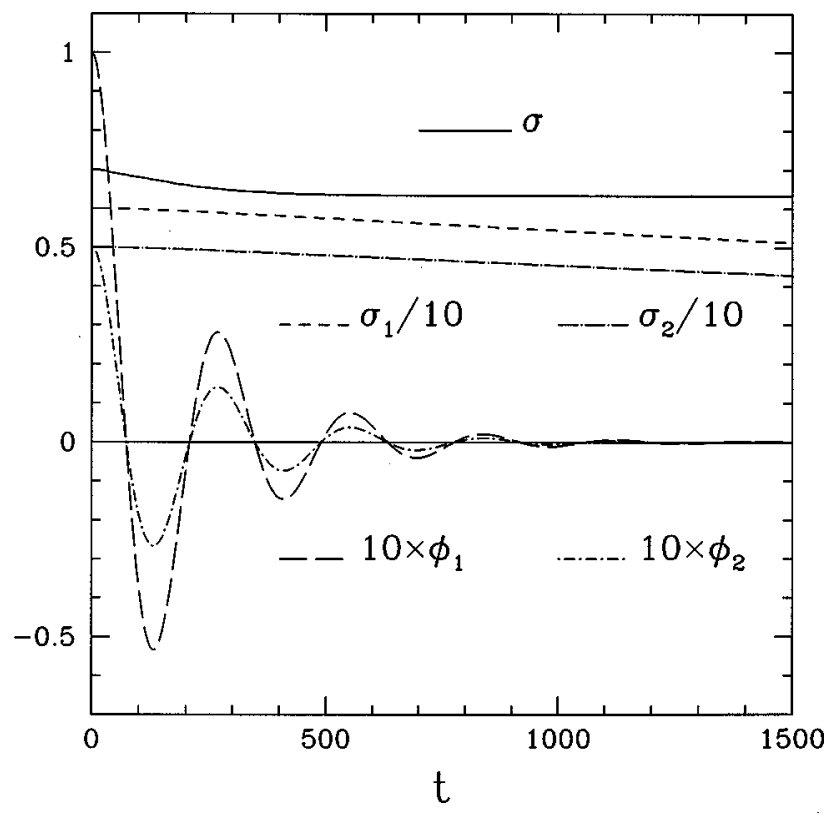

FIG. 2. Evolution of $\sigma, \sigma_{1}, \sigma_{2}, \phi_{1}, \phi_{2}$ (in units of $m_{\mathrm{Pl}}$ ) for the theory of Eq. (3.1) with $\mu / m_{\mathrm{Pl}}=5 \times 10^{-4}, \lambda=0.05, \mathrm{~m} / \mathrm{m}_{\mathrm{Pl}}$ $=1 \times 10^{-3}$, and initial conditions $\sigma_{0} / m_{\mathrm{Pl}}=0.7,\left[\phi_{1}\right]_{0} / m_{\mathrm{Pl}}=0.1$, $\left[\phi_{2}\right]_{0} / m_{\mathrm{Pl}}=0.05,\left[\sigma_{1}\right]_{0} / m_{\mathrm{Pl}}=6,\left[\sigma_{2}\right]_{0} / m_{\mathrm{Pl}}=5$.

where $S^{\prime}$ is a gauge singlet chiral superfield, whose scalar components are given by $S^{\prime}=\left(\sigma_{1}+i \sigma_{2}\right) / \sqrt{2}$. This is a very simple extension of the minimal superpotential giving rise to hybrid inflation. The above superpotential is the only renormalizable one consistent with a continuous $\mathrm{U}(1) R$ symmetry under which $W \rightarrow e^{i \theta} W, S \rightarrow e^{i \theta} S, S^{\prime} \rightarrow e^{i \theta / 2} S^{\prime}, \Phi \Phi \rightarrow \Phi \Phi$. The potential derived from $W$ is given by Eq. (2.4) with the addition of the mass term

$$
\Delta V\left(\sigma_{1}, \sigma_{2}\right)=\frac{1}{2} m^{2}\left(\sigma_{1}^{2}+\sigma_{2}^{2}\right)
$$

For initial values of $\sigma_{1}$ and $\sigma_{2}$ larger than $m_{\mathrm{Pl}}$, this system undergoes a first stage of chaotic inflation, followed by a second stage of hybrid inflation similar to the one discussed in the previous section. In Fig. 2 we present the evolution of the various fields for the choice of parameters $\mu / m_{\mathrm{Pl}}$ $=5 \times 10^{-4}, \lambda=0.05, \mathrm{~m} / \mathrm{m}_{\mathrm{Pl}}=10^{-3}$. The initial values of the fields have been taken to be $\sigma_{0} / m_{\mathrm{Pl}}=0.7,\left[\phi_{1}\right]_{0} / m_{\mathrm{Pl}}=0.1$, $\left[\phi_{2}\right]_{0} / m_{\mathrm{Pl}}=0.05,\left[\sigma_{1}\right]_{0} / m_{\mathrm{Pl}}=6,\left[\sigma_{2}\right]_{0} / m_{\mathrm{Pl}}=5$, with their time derivatives equal to zero. We observe that the fields $\sigma_{1}, \sigma_{2}$ quickly approach a slow-roll regime and they attain almost constant velocities. This regime terminates when $\sigma_{1,2} \simeq 1$. During this time, the vacuum energy density is dominated by the contribution of Eq. (3.2) and a period of inflation takes place. The Hubble parameter has a value $H_{i} / m_{\mathrm{Pl}} \simeq 3.4 \times 10^{-3}$ at the beginning of the slow-roll regime, which drops to $H_{f} / m_{\mathrm{PI}} \simeq 6 \times 10^{-4}$ at its end. A total number of $N_{1} \simeq 16 e$-foldings is generated by this first stage of inflation.

In the model we have described, the onset of inflation occurs "naturally." The initial correlation lengths of the various fields are comparable to the Hubble length $H^{-1}$ and, therefore, an initial field configuration homogeneous over a few Hubble lengths is not unlikely.

The evolution of $\sigma, \phi_{1}, \phi_{2}$ is also depicted in Fig. 2. The presence of a large "friction" term $H \sim m \sqrt{\sigma_{1}^{2}+\sigma_{2}^{2}} / m_{\mathrm{Pl}}$ in their evolution equations forces these fields to quickly settle down along their flat direction. The time derivatives of $\phi_{1}$, $\phi_{2}$ go to zero, while that of $\sigma$ reaches a very small value because of the small slope induced by the contribution of Eq. (2.8). The total classical evolution of $\sigma$ during the first stage of inflation is negligible. This behavior should be contrasted with the one depicted in Fig. 1. In that case the "friction" term $H \sim \mu^{2} / m_{\mathrm{PI}}$ was smaller by several orders of magnitude and the field oscillations decayed very slowly.

After the end of the first stage of inflation, an intermediate stage takes place during which $\sigma_{1}$ and $\sigma_{2}$ oscillate around zero. The energy density of the Universe decreases, until the point where the final vacuum energy density $\mu^{4}$ starts to dominate and the second stage of inflation begins. During all this time, the system $\left(\sigma, \phi_{1,2}\right)$ remains unperturbed at the classical level, as it is not coupled to $\sigma_{1}, \sigma_{2}$. However, a more careful analysis is required in order to take into account quantum fluctuations of the fields.

During the first stage of inflation, the almost massless field $\sigma$ has a spectrum of quantum-mechanical fluctuations characterized by

$$
(\Delta \sigma)_{k}^{2}=\left(\frac{H_{1}}{2 \pi}\right)^{2}
$$

where $H_{1}$ is the Hubble parameter. For constant $H_{1}$, the freezing of fluctuations that cross the horizon results in a mean-square fluctuation of the classical field $\sigma$ [23],

$$
(\Delta \sigma)^{2}=N_{1}\left(\frac{H_{1}}{2 \pi}\right)^{2}
$$

where $N_{1}$ is the number of $e$-foldings. For the model we are discussing, $H_{1}$ is slowly decreasing, and the above expression gives an upper bound on $\Delta \sigma$ if the initial value $H_{i}$ is employed. For $N_{1} \simeq 16, H_{1}=H_{i} \simeq 3.4 \times 10^{-3} m_{\mathrm{Pl}}$, we conclude that the mean-square fluctuation of $\sigma$ is negligible compared to its mean value $\sigma \simeq 0.63$. At the end of the first stage of inflation, the energy density in spatial gradient terms associated with the perturbations $\sim H_{f} / 2 \pi$ of massless fields like $\sigma$ is $\sim H_{f}^{4} / 4 \pi^{2}$. It is further reduced by the subsequent expansion and stays much smaller than the total energy density during the whole intermediate stage between the two inflations. As a result, it is negligible compared to the vacuum energy density $\mu^{4}$ when this begins to dominate.

The fluctuations of the massive fields $\phi_{i}, i=1,2$, generated by the first stage of inflation are given by

$$
\left(\Delta \phi_{i}\right)_{k}^{2}=\left(c \frac{H_{1}^{2}}{m_{i}}\right)^{2},
$$

where $m_{i}$ is their mass and $c=\mathcal{O}\left(10^{-1}\right)$. During the intermediate stage, the amplitude of these fluctuations drops 
$\sim R^{-3 / 2}$. As a result, we expect that, when the energy density becomes comparable to $\mu^{4}$, this amplitude is approximately given by

$$
\left(\Delta \phi_{i}\right)_{k} \simeq c \frac{H_{1}^{2}}{m_{i}}\left(\frac{H_{2}}{H_{1}}\right)^{1 /(1+w)} .
$$

Here $H_{1}$ has to be taken close to its value towards the end of the first stage of inflation, $H_{1} \simeq H_{f}$. Here $H_{2}$ is the Hubble parameter during the second stage, and $w$ is determined by the dynamics of the intermediate stage. For a system of massive oscillating fields or a matter-dominated universe, $w$ $=0$. For a radiation-dominated universe, $w=1 / 3$. For the model we are considering, $H_{2} / m_{\mathrm{Pl}}=1.4 \times 10^{-7}$ and the fluctuations of $\phi_{1}, \phi_{2}$ are much smaller than the bound of Eq. (2.17).

We conclude that the necessary conditions for the onset of the second stage of inflation are "naturally" satisfied. Because of the expansion during the first stage, the homogeneous regions extend far beyond the Hubble length $\mathrm{H}_{2}^{-1}$. The fields $\sigma, \phi_{1}, \phi_{2}$ are localized on the flat direction of the potential. More specifically, the degree of homogeneity of $\phi_{1}, \phi_{2}$ satisfies the constraint of Eq. (2.17). Thus the second stage of inflation "naturally" sets in. It generates $N_{2}$ $\simeq 6.3 \times 10^{3} \mathrm{e}$-foldings and density perturbations in agreement with the COBE observations.

\section{B. Hybrid-hybrid model}

The model described by the superpotential

$W=S\left(-\mu^{2}+\lambda \bar{\Phi} \Phi\right)+S^{\prime}\left(-\mu^{\prime 2}+\lambda^{\prime} \bar{\Psi} \Psi+g \bar{\Phi} \Phi\right)$

is essentially composed of two sectors similar to the one of Eq. (2.1). We assume that the new superfields $\Psi, \bar{\Psi}$ transform under an internal U(1) gauge symmetry. The $S^{\prime}$ superfield is a gauge singlet. Under the continuous $\mathrm{U}(1) R$ symmetry, the new superfields transform as $S^{\prime} \rightarrow e^{i \theta} S^{\prime}, \bar{\Psi} \Psi$ $\rightarrow \Psi \Psi$. The absence of a coupling $S \Psi \Psi$ is explained if we regard $S$ as the linear combination of the gauge singlets that does not couple to $\bar{\Psi} \Psi$.

Making use of the continuous symmetries of $W$ and staying along the $D$-flat directions, we can choose the real components of the various canonically normalized scalar fields as

$$
\begin{gathered}
S=\frac{\sigma}{\sqrt{2}}, \quad \Phi=\bar{\Phi}=\frac{\phi_{1}+i \phi_{2}}{2}, \\
S^{\prime}=\frac{\sigma_{1}+i \sigma_{2}}{\sqrt{2}}, \quad \Psi=\bar{\Psi}=\frac{\psi_{1}+i \psi_{2}}{2} .
\end{gathered}
$$

The potential is then given by the expression

$$
\begin{aligned}
V= & \mu^{\prime 4}-\frac{g}{2} \mu^{\prime 2}\left(\phi_{1}^{2}-\phi_{2}^{2}\right)+\frac{g^{2}}{16}\left(\phi_{1}^{2}+\phi_{2}^{2}\right)^{2}-\frac{\lambda^{\prime}}{2} \mu^{\prime 2}\left(\psi_{1}^{2}-\psi_{2}^{2}\right)+\frac{\lambda^{\prime 2}}{16}\left(\psi_{1}^{2}+\psi_{2}^{2}\right)^{2}+\frac{\lambda^{\prime} g}{2}\left[\frac{1}{4}\left(\phi_{1}^{2}-\phi_{2}^{2}\right)\left(\psi_{1}^{2}-\psi_{2}^{2}\right)\right. \\
& \left.+\phi_{1} \phi_{2} \psi_{1} \psi_{2}\right]+\mu^{4}-\frac{\lambda}{2} \mu^{2}\left(\phi_{1}^{2}-\phi_{2}^{2}\right)+\frac{\lambda^{2}}{16}\left(\phi_{1}^{2}+\phi_{2}^{2}\right)^{2}+\frac{\lambda^{\prime 2}}{4}\left(\sigma_{1}^{2}+\sigma_{2}^{2}\right)\left(\psi_{1}^{2}+\psi_{2}^{2}\right)+\frac{1}{4}\left[\left(\lambda \sigma+g \sigma_{1}\right)^{2}+g^{2} \sigma_{2}^{2}\right]\left(\phi_{1}^{2}+\phi_{2}^{2}\right),
\end{aligned}
$$

where the mass scales $\mu^{\prime}, \mu$ are chosen to satisfy the inequality $\mu^{\prime} \gg \mu$. The minima of this potential are located at

$$
\begin{array}{ll}
\sigma=0, & \phi_{1}^{2}=\frac{4}{\lambda} \mu^{2}, \quad \phi_{2}=0, \\
\sigma_{1}=\sigma_{2}=0, \quad \psi_{1}^{2}=\frac{4}{\lambda^{\prime}} \mu^{\prime 2}-\frac{4 g}{\lambda \lambda^{\prime}} \mu^{2} \simeq \frac{4}{\lambda^{\prime}} \mu^{\prime 2}, \quad \psi_{2}=0 .
\end{array}
$$

For $\phi_{1}=\phi_{2}=\psi_{1}=\psi_{2}=0$, the potential is independent of $\sigma, \sigma_{1}, \sigma_{2}$ and has the value $V=\mu^{\prime 4}+\mu^{4} \simeq \mu^{\prime 4}$, which is the vacuum energy density during the first stage of inflation. The mass terms of the $\phi_{1,2}$ and $\psi_{1,2}$ fields are

$$
\begin{aligned}
{\left[M_{\phi}^{2}\right]_{1,2} } & =\mp g \mu^{\prime 2} \mp \lambda \mu^{2}+\frac{1}{2}\left[\left(\lambda \sigma+g \sigma_{1}\right)^{2}+g^{2} \sigma_{2}^{2}\right] \\
& \simeq \mp g \mu^{\prime 2}+\frac{1}{2}\left[\left(\lambda \sigma+g \sigma_{1}\right)^{2}+g^{2} \sigma_{2}^{2}\right] \\
{\left[M_{\psi}^{2}\right]_{1,2} } & =\mp \lambda^{\prime} \mu^{\prime 2}+\frac{\lambda^{\prime 2}}{2}\left(\sigma_{1}^{2}+\sigma_{2}^{2}\right) .
\end{aligned}
$$

We see that the squared mass term of the $\psi_{1}$ field becomes negative for

$$
\sigma_{1}^{2}+\sigma_{2}^{2}<\frac{2 \mu^{\prime 2}}{\lambda^{\prime}}
$$

while that of the $\phi_{1}$ field turns negative for

$$
\left(\sigma_{1}+\frac{\lambda}{g} \sigma\right)^{2}+\sigma_{2}^{2}<\frac{2 \mu^{\prime 2}}{g}
$$

signaling instabilities in the $\psi_{1}$ and $\phi_{1}$ directions. 


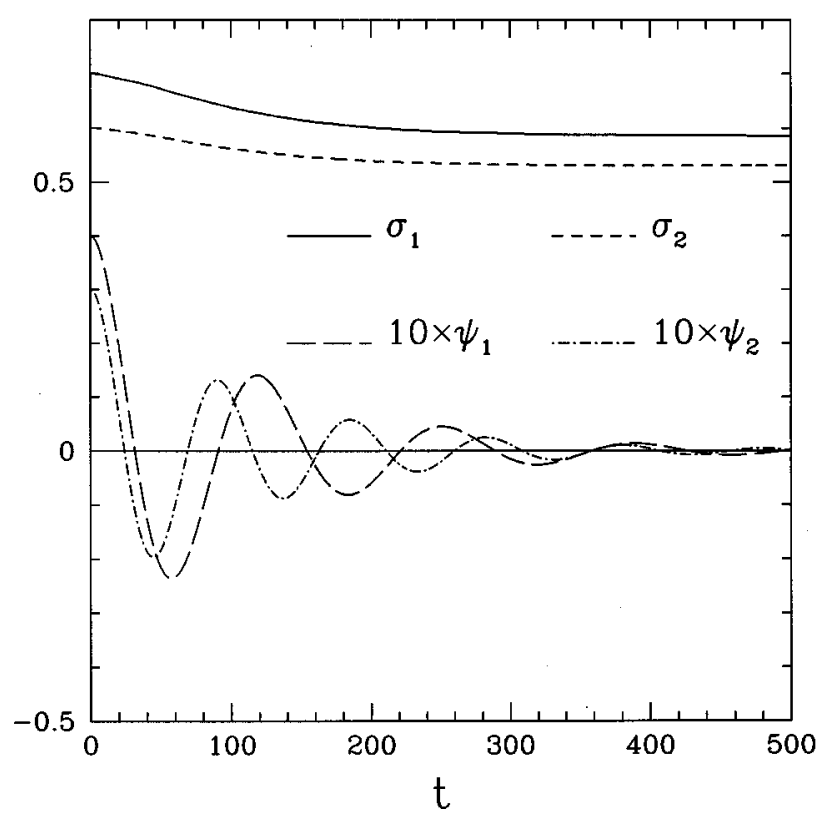

FIG. 3. Evolution of $\sigma_{1}, \sigma_{2}, \psi_{1}, \psi_{2}$ (in units of $m_{\mathrm{Pl}}$ ) for the theory of Eq. (3.7) with $\mu / m_{\mathrm{Pl}}=5 \times 10^{-4}, \lambda=0.05, \mu^{\prime} / m_{\mathrm{Pl}}=0.1$, $\lambda^{\prime}=0.1, g=0.1$, and initial conditions $\sigma_{0} / m_{\mathrm{Pl}}=0.7,\left[\phi_{1}\right]_{0} / m_{\mathrm{Pl}}$ $=0.1, \quad\left[\phi_{2}\right]_{0} / m_{\mathrm{Pl}}=0.05, \quad\left[\sigma_{1}\right]_{0} / m_{\mathrm{Pl}}=0.7, \quad\left[\sigma_{2}\right]_{0} / m_{\mathrm{Pl}}=0.6$, $\left[\psi_{1}\right]_{0} / m_{\mathrm{Pl}}=0.04,\left[\psi_{2}\right]_{0} / m_{\mathrm{Pl}}=0.03$.

The flatness of the potential is lifted by radiative corrections. For $\sigma$ and $\sigma_{1,2}$ far above the instability points, the one-loop contribution to the effective potential is

$$
\begin{aligned}
\Delta V\left(\sigma, \sigma_{1}, \sigma_{2}\right) \simeq & \frac{g^{2}}{16 \pi^{2}} \mu^{\prime 4}\left[\ln \left(\frac{\left(\lambda \sigma+g \sigma_{1}\right)^{2}+g^{2} \sigma_{2}^{2}}{2 \Lambda^{2}}\right)+\frac{3}{2}\right] \\
& +\frac{\lambda^{\prime 2}}{16 \pi^{2}} \mu^{\prime 4}\left[\ln \left(\frac{\lambda^{\prime 2}\left(\sigma_{1}^{2}+\sigma_{2}^{2}\right)}{2 \Lambda^{2}}\right)+\frac{3}{2}\right]
\end{aligned}
$$

The second stage of inflation can take place for $\phi_{1}=\phi_{2}$ $=\sigma_{1}=\sigma_{2}=\psi_{2}=0, \psi_{1}^{2}=4 \mu^{\prime 2} / \lambda^{\prime}$. Then the potential is independent of $\sigma$ and the vacuum energy density is $V=\mu^{4}$. A small slope in the $\sigma$ direction is provided by the radiative contribution of Eq. (2.8), leading to a second stage of inflation in complete analogy to the one we discussed in Sec. II.

In Figs. 3 and 4 we present the evolution of the various fields for the model of Eq. (3.7) with $\mu / m_{\mathrm{Pl}}=5 \times 10^{-4}, \lambda$ $=0.05, \mu^{\prime} / m_{\mathrm{Pl}}=0.1, \lambda^{\prime}=0.1, g=0.1$, and initial conditions $\sigma_{0} / m_{\mathrm{Pl}}=0.7,\left[\phi_{1}\right]_{0} / m_{\mathrm{Pl}}=0.1,\left[\phi_{2}\right]_{0} / m_{\mathrm{Pl}}=0.05,\left[\sigma_{1}\right]_{0} / m_{\mathrm{Pl}}$ $=0.7,\left[\sigma_{2}\right]_{0} / m_{\mathrm{Pl}}=0.6,\left[\psi_{1}\right]_{0} / m_{\mathrm{Pl}}=0.04,\left[\psi_{2}\right]_{0} / m_{\mathrm{Pl}}=0.03$. The initial time derivatives of the fields have been taken equal to zero. We observe that the system quickly settles along the flat direction $\phi_{1}=\phi_{2}=\psi_{1}=\psi_{2}=0$ and a first stage of inflation begins. As the initial correlation lengths of the various fields are comparable to the initial Hubble length $H^{-1}$, it is not unlikely to have a field configuration homogeneous over a few Hubble lengths. The initial homogeneity is

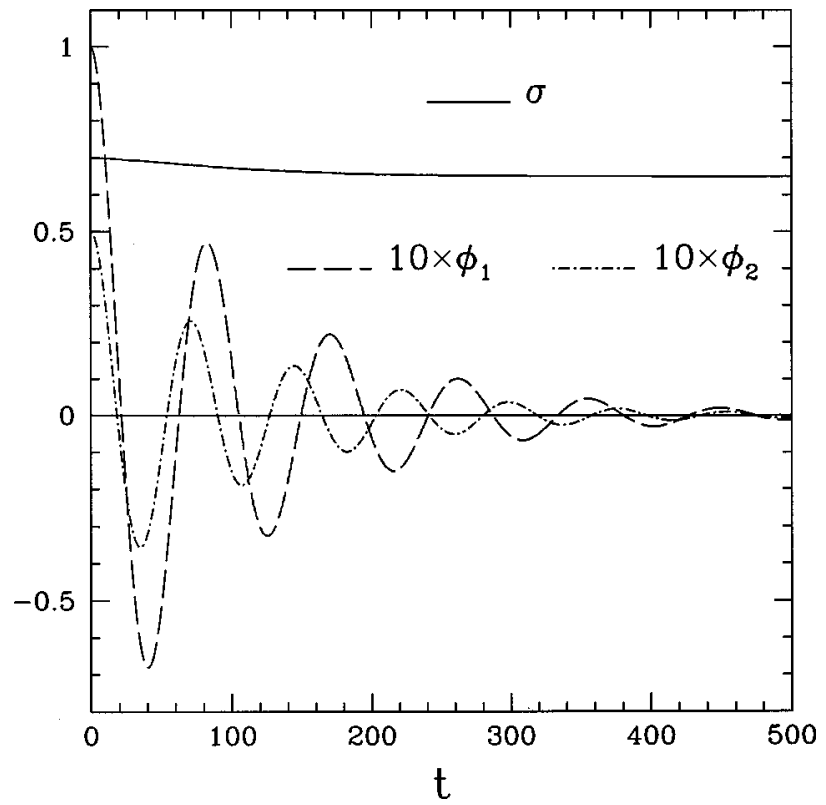

FIG. 4. The evolution of $\sigma, \phi_{1}, \sigma_{2}$ (in units of $m_{\mathrm{PI}}$ ) for the theory of Fig. 3.

expected to be preserved by the short evolution to the flat direction. As a result, the onset of inflation occurs "naturally' in this model.

The first stage of inflation is characterized by a constant Hubble parameter $H_{1} / m_{\mathrm{Pl}}=5.8 \times 10^{-3}$. It lasts until an instability point is reached. For our choice of couplings, the mass term of the $\psi_{1}$ field is the first one to turn negative. This occurs for $\sigma / m_{\mathrm{Pl}}=0.61, \sigma_{1} / m_{\mathrm{Pl}}=0.32, \sigma_{2} / m_{\mathrm{Pl}}=0.31$. A total number of $N_{1} \simeq 1.5 \times 10^{3} e$-foldings is generated by the first stage of inflation. The subsequent evolution proceeds in complete analogy to the discussion in the previous section. An intermediate stage takes place, during which the fields $\sigma_{1,2}, \psi_{1,2}$ oscillate around the minimum at $\sigma_{1}=\sigma_{2}=\psi_{2}=0$, $\psi_{1}^{2}=4 \mu^{\prime 2} / \lambda^{\prime}$, and the energy density is dissipated through expansion. When it becomes $\sim \mu^{4}$ the second stage of inflation begins, with a constant Hubble parameter $H_{2} / m_{\mathrm{Pl}}=1.4$ $\times 10^{-7}$. Because of the expansion during the first stage, the homogeneous regions extend far beyond the Hubble length $H_{2}^{-1}$. Moreover, the fields $\sigma, \phi_{1}, \phi_{2}$ are localized on the flat direction of the potential. The amplitudes of $\phi_{1}, \phi_{2}$ are given by Eq. (3.6) and satisfy the constraint of Eq. (2.17). Thus the second stage of inflation "naturally" sets in. It generates $N_{2} \simeq 5.9 \times 10^{3} e$-foldings and density perturbations in agreement with the COBE observations.

\section{CONCLUSIONS}

In this paper we addressed the problem of fine-tuning of the initial conditions for supersymmetric hybrid inflation. This problem is generated by the difference between the energy scale at which the Universe emerges from the Planck era (near $m_{\mathrm{Pl}}$ ) and the inflationary scale implied by the COBE observations $\left(V^{1 / 4} \sim 10^{-3} m_{\mathrm{Pl}}\right)$. We suggested a simple resolution of the issue of fine-tuning by considering a scenario with two stages of inflation at two different energy 
scales. The first stage has a typical scale not far from $m_{\mathrm{Pl}}$. Consequently, the Hubble parameter stays large until the fields settle down along the direction that produces inflation. Because of the large "friction" term in the equations of motion, the initial part of the evolution towards the inflationary direction is short and the first stage of inflation occurs "naturally." After the end of this stage, a subset of the fields moves towards a minimum of the potential, around which it performs damped oscillations. During this intermediate stage, the energy density is reduced through expansion. A second stage of inflation begins when the energy density falls below the false vacuum energy density associated with a second-order phase transition involving the remaining fields. The homogeneity far beyond the Hubble length that was produced during the first inflationary stage makes the onset of the second stage "natural," despite the fact that the Hubble length is much larger than the one of the first stage. The second stage of inflation generates the density perturbations that result in the cosmic microwave background anisotropy observed by COBE. In Sec. III we gave two realizations of the above scenario in the context of global supersymmetry. The first employs one chaotic and one hybrid inflation, while the second one employs two hybrid inflations that take place for field values below $m_{\mathrm{Pl}}$.

The generalization of the two-stage inflationary scenario in the context of supergravity is difficult. The case of a firststage chaotic inflation suffers from the obvious difficulty that it requires field values larger than $m_{\mathrm{Pl}}$. For the second scenario that involves two hybrid inflations, the tree-level potential has a multiple $F$-flat direction at $\Phi=\Phi=\Psi=\bar{\Psi}=0$. When global $N=1$ supersymmetry is replaced by $N=1 \mathrm{su}-$ pergravity, all flat directions are, in general, lifted and inflation becomes impossible. In the context of canonical supergravity (minimal Kähler potential) and a linear superpotential of the form $W=-\mu^{2} S$ (like the one encountered in hybrid inflationary models), a miraculous cancellation takes place that prevents the appearance of a mass term for $S$ [2]. Therefore, there is a possibility for $S$ to play the role of the inflaton. However, for a superpotential such as the one of Eq. (3.7), only a linear combination of $S$ and $S^{\prime}$ stays "massless," with the orthogonal combination acquiring a large mass term [6]. This implies that only one inflationary stage is likely to survive in the context of supergravity. However, this argument does not apply to the case where inflation is driven by a $D$-term energy density [7]. The two-stage inflationary scenario may then be possible along an appropriate combination of $F$-flat and $D$-flat directions.

\section{ACKNOWLEDGMENTS}

We would like to thank G. Dvali, J. Garcia-Bellido, and G. Lazarides for useful discussions. This research was supported by the E.U. under TMR Contract No. ERBFMRXCT96-0090.
[1] A. D. Linde, Phys. Lett. B 259, 38 (1991); Phys. Rev. D 49, 748 (1994).

[2] E. J. Copeland, A. R. Liddle, D. H. Lyth, E. D. Stewart, and D. Wands, Phys. Rev. D 49, 6410 (1994).

[3] G. Dvali, Q. Shafi, and R. Schaefer, Phys. Rev. Lett. 73, 1886 (1994).

[4] E. D. Stewart, Phys. Rev. D 51, 6847 (1995).

[5] G. Lazarides and C. Panagiotakopoulos, Phys. Rev. D 52, 559 (1995).

[6] C. Panagiotakopoulos, Phys. Rev. D 55, 7335 (1997); Phys. Lett. B 402, 257 (1997).

[7] P. Binétruy and G. Dvali, Phys. Lett. B 388, 241 (1996); E. Halyo, ibid. 387, 43 (1996).

[8] A. D. Linde and A. Riotto, Phys. Rev. D 56, 1841 (1997).

[9] S. Dimopoulos, G. Dvali, and R. Rattazzi, Phys. Lett. B 410, 119 (1997).

[10] D. S. Goldwirth and T. Piran, Phys. Rep. 214, 223 (1992).

[11] A. Albrecht and P. J. Steinhardt, Phys. Rev. Lett. 48, 1220 (1982); A. D. Linde, Phys. Lett. 114B, 431 (1982); 166B, 335, 340 (1982).

[12] A. A. Starobinsky, Phys. Lett. 117B, 175 (1982).

[13] A. D. Linde, Phys. Lett. 162B, 281 (1985).
[14] A. D. Linde, Phys. Lett. 129B, 177 (1983); Particle Physics and Inflationary Cosmology (Harwood Academic, Chur, Switzerland, 1990).

[15] G. Lazarides, C. Panagiotakopoulos, and N. D. Vlachos, Phys. Rev. D 54, 1369 (1996).

[16] G. Lazarides and N. D. Vlachos, Phys. Rev. D 56, 4562 (1997).

[17] N. Tetradis, Phys. Rev. D 57, 5997 (1998).

[18] S. W. Hawking, Phys. Lett. 115B, 295 (1982); A. H. Guth and S.-Y. Pi, Phys. Rev. Lett. 49, 1110 (1982); D. H. Lyth, Phys. Lett. 147B, 403 (1984); 150B, 465 (1985); Phys. Rev. D 31, 1792 (1985).

[19] A. R. Liddle and D. H. Lyth, Phys. Rep. 231, 1 (1993).

[20] D. H. Lyth, Report No. LANCS-TH/9614, hep-ph/9609431.

[21] A. D. Linde, Phys. Lett. B 202, 194 (1988).

[22] K.-I. Izawa, M. Kawasaki, and T. Yanagida, Phys. Lett. B 411, 249 (1997).

[23] T. S. Bunch and P. C. W. Davies, Proc. R. Soc. London A360, 117 (1978); A. Vilenkin and L. Ford, Phys. Rev. D 26, 1231 (1982); A. D. Linde, Phys. Lett. 116B, 335 (1982); A. Vilenkin, Nucl. Phys. B226, 527 (1983). 